\title{
PRODUCTS OF AUTOMATA AND THE PROBLEM OF COVERING
}

\author{
BY \\ ABRAHAM GINZBURG AND MICHAEL YOELI
}

Introduction. The first part of this paper (Theorems 1-3) gives a short, unified treatment of (Mealy type [12]) automata (sequential machines). By associating with every input two binary relations ("next-state" and "output" relations) we obtain an easy and concise algebraic method for the description and study of complete or partial, finite or infinite automata.

In the second part (Theorems 4-7) we develop further the algebraic decomposition theory of automata, continuing previous work by J. Hartmanis [7]-[9] and M. Yoeli [16]-[18]. To make the exposition self-contained, we repeat some of the material contained in [18]. For other approaches to automata decompositions the reader is referred to [1], [3], [5], [10], [11].

In [18] the concept of semi-automaton (see Section I) was introduced and methods for its decomposition by means of overlapping partitions were derived. In the present paper these investigations are extended to (Mealy type) automata and the problems of covering specified automata by direct and cascade products are studied.

This approach leads to an interesting new algebraic concept, namely that of a weak (i.e., generalized) homomorphism defined by overlapping partitions. Recently this concept and its applicability to partial algebras has been further investigated [19] and generalizations of well-known results on homomorphisms and subdirect products of partial algebras have been obtained.

We hope that this mutual enrichment of pure algebra and automata theory will be of interest to both the applied as well as the pure algebraist. The newcomer to automata theory is referred to the introductory texts [2], [4]-[6] and the collection of papers [13], [14]. [13] also contains a very extensive bibliography on sequential machines.

The contribution of the second author was supported by the U. S. Office of Naval Research, Information Systems Branch, under Contract No. $\mathrm{N}$ 62558-3510. The present paper is a revised version of Technical Report No. 15, Hebrew University, Jerusalem, Israel (July, 1963), DDCDocument AD-417 380 .

I. Preliminaries. We first recall some basic concepts on binary relations to be used in the sequel.

Received by the editors December 5, 1963. 
Let $R$ be a relation between the sets $M$ and $N$, i.e., $R \subseteq M \times N$. Following [15] we denote

$$
\mid R=\{m \mid \exists n:(m, n) \in R\} \text { and } R \mid=\{n \mid \exists m:(m, n) \in R\} .
$$

For $M^{\prime} \subseteq M, M^{\prime} R=\left\{n \mid \exists m \in M^{\prime}:(m, n) \in R\right\}$.

The following is evident:

$$
\begin{aligned}
& R_{1} \subseteq R_{2} \Rightarrow R_{1} R \subseteq R_{2} R, R R_{1} \subseteq R R_{2}, R_{1}^{-1} \subseteq R_{2}^{-1}, \\
& \mid R=M \Rightarrow R R^{-1} \supseteq I_{M}=\{(m, m) \mid m \in M\} .
\end{aligned}
$$

Next we list the following basic definitions concerning automata.

We define a semi-automaton $A$ as a system $\left\langle S_{A}, X_{A}, \Delta^{A}\right\rangle$ where $S_{A}$ is a nonempty set (of states), $X_{A}$ is a nonempty set (of inputs) and $\Delta^{A}$ (the next-state function) a mapping from a subset of $S_{A} \times X_{A}$ into $S_{A}$.

With every $x \in X_{A}$ we associate a binary relation $\bar{x}^{A}$ over $S_{A}$ defined by:

$$
(s, t) \in \bar{x}^{A} \Leftrightarrow \Delta^{A}(s, x)=t .
$$

Clearly the semi-automaton $A$ can alternatively be defined as a triple $\left\langle S_{A}, X_{A},\left\{\bar{x}^{A} \mid x \in X_{A}\right\}\right\rangle$ where the $\bar{x}^{A}$ are mappings from $S_{A}$ into $S_{A}$.

The following definitions are taken over from [18]. A decomposition $\pi$ of a given set $S$ is a family of nonempty subsets of $S$ whose set union is $S$.

Let $A=\left\langle S_{A}, X_{A}, \Delta^{A}\right\rangle$ be a semi-automaton and $\pi$ a decomposition of $S_{A} \cdot \pi$ is admissible by $A$, if for every $H \in \pi$ and every $x \in X_{A}$ there exists a $K \in \pi$ such that $H \bar{x}^{A} \subseteq K$. The semi-automaton $B=\left\langle S_{B}, X_{B}, \Delta^{B}\right\rangle$ is a $\pi$-factor of $A$ if (i) $S_{B}=\pi$, (ii) $X_{A}=X_{B}$, (iii) for every $H \in \pi$ and every $x \in X_{A}, H \bar{x}^{A}=\emptyset$ implies $H \bar{x}^{B}=\emptyset$ and (iv) for every $H \in \pi$ and every $x \in X_{A}, H \bar{x}^{A} \subseteq H \bar{x}^{B}$.

Clearly, if $A=\left\langle S_{A}, X_{A}, \Delta^{A}\right\rangle$ is a semi-automaton and $\pi$ a partition of $S_{A}$ admissible by $A$, there exists exactly one $\pi$-factor $B$ of $A$ (notation: $B=A / \pi)$.

The direct product $A \times B$ of the semi-automata $A=\left\langle S_{A}, X, \Delta^{A}\right\rangle$ and $B=\left\langle S_{B}, X, \Delta^{B}\right\rangle$ is the semi-automaton $C=\left\langle S_{C}, X, \Delta^{C}\right\rangle$ where $S_{C}=S_{A} \times S_{B}$ and $\left(s_{A}, s_{B}\right) \bar{x}^{C}=s_{A} \bar{x}^{A} \times s_{B} \bar{x}^{B}$.

Anautomaton $\hat{A}=\left\langle S_{A}, X_{A}, Z_{A}, \Delta^{A}, \Lambda^{A}\right\rangle$ is a semi-automaton $A=\left\langle S_{A}, X_{A}, \Delta^{A}\right\rangle$ together with a nonempty set (of outputs) $Z_{A}$ and a mapping $\Lambda^{A}$ (the output function) from a subset of $S_{A} \times X_{A}$ into $Z_{A}$.

As previously, we associate with each input $x \in X_{A}$ a binary relation $x_{*}^{A} \subseteq S_{A} \times Z_{A}$ defined by:

$$
(s, z) \in x_{*}^{A} \Leftrightarrow \Lambda^{A}(s, x)=z .
$$

The semi-automaton $A$ of the automaton $\hat{A}$ is complete if $\Delta^{A}$ is completely defined, i.e., for every $s \in S_{A}$ and every $x \in X_{A}$. The automaton $\hat{A}$ is complete if $A$ is complete and $\Lambda^{A}$ is also completely defined. 
As usual, we extend our considerations to input tapes $\xi=x_{1} x_{2} \cdots x_{K}$, $x_{i} \in X_{A}$. To each such tape $\xi$ there correspond the following binary relations:

$$
\begin{aligned}
& \bar{\xi}^{A}=\bar{x}_{1}^{A} \bar{x}_{2}^{A} \cdots \bar{x}_{K}^{A}, \\
& \xi_{*}^{A}=\bar{x}_{1}^{A} \bar{x}_{2}^{A} \cdots \bar{x}_{K-1}^{A} x_{K *}^{A} .
\end{aligned}
$$

The right-hand sides of the above formulas are the relational products of the corresponding relations. $\bar{\xi}^{A}$ gives the state transformation due to the tape $\xi$, and $\xi_{*}^{A}$ indicates the last output due to $\xi$, both for any initial state.

For a partial automaton $\hat{A}$ and a certain tape $\xi, \bar{\xi}^{A}$ or $\xi_{*}^{A}$ can be the empty relation $\emptyset$.

\section{Homomorphisms of automata.}

DEFINITION 1. Given two automata

$$
\hat{A}=\left\langle S_{A}, X_{A}, Z_{A}, \Delta^{A}, \Lambda^{A}\right\rangle
$$

and

$$
\hat{B}=\left\langle S_{B}, X_{B}, Z_{B}, \Delta^{B}, \Lambda^{B}\right\rangle
$$

with $X_{A}=X_{B}$ and $Z_{A}=Z_{B}$, the mapping $\phi$ of $S_{A}$ onto $S_{B}$ is a homomorphism of $\hat{A}$ onto $\hat{B}$ if for every $x \in X_{A}=X_{B}$

$$
\begin{aligned}
& \text { (i) } \bar{x}^{A} \phi \subseteq \phi \bar{x}^{B} \text {, } \\
& \text { (ii) } x_{*}^{A} \subseteq \phi x_{*}^{B} .
\end{aligned}
$$

Note. $\bar{x}^{A} \phi$ denotes the relational product of $\bar{x}^{A}$ and $\phi\left(\subseteq S_{A} \times S_{B}\right)$, etc.

Definition 2. Given two automata $\hat{A}$ and $\hat{B}$ as in Definition 1 , and a binary relation $\psi$ with $\mid \psi=S_{A}$ and $\psi \mid=S_{B}$, the relation $\psi$ is a weak homomorphism of $\hat{A}$ onto $\hat{B}$, if for every $x \in X_{A}=X_{B}$

$$
\text { (i) } \psi^{-1} \bar{x}^{A} \subseteq \bar{x}^{B} \psi^{-1} \text {, }
$$$$
\text { (ii) } \psi^{-1} x_{*}^{A} \subseteq x_{*}^{B} \text {. }
$$

If $\psi$ in Definition 2 is a mapping of $S_{A}$ onto $S_{B}$ then the conditions (1) and (2) are equivalent. Indeed, the assumptions $\mid \psi=S_{A}$ and $\psi \mid=S_{B}$ imply $\psi \psi^{-1} \supseteq I_{S_{A}}$ and $\psi^{-1} \psi \supseteq I_{S_{B}}$.

The further assumption that $\psi$ is a mapping leads to $\psi^{-1} \psi=I_{S_{B}}$. Now,

$$
\bar{x}^{A} \psi \subseteq \psi \bar{x}^{B} \Rightarrow \psi^{-1} \bar{x}^{A} \psi \psi^{-1} \subseteq \psi^{-1} \psi \bar{x}^{B} \psi^{-1} \Rightarrow \psi^{-1} \bar{x}^{A} \subseteq \bar{x}^{B} \psi^{-1}
$$

and

$$
x_{*}^{A} \subseteq \psi x_{*}^{B} \Rightarrow \psi^{-1} x_{*}^{A} \subseteq \psi^{-1} \psi x_{*}^{B}=x_{*}^{B} .
$$

Conversely, 


$$
\psi^{-1} \bar{x}^{A} \subseteq \bar{x}^{B} \psi^{-1} \Rightarrow \psi \psi^{-1} \bar{x}^{A} \psi \subseteq \psi \bar{x}^{B} \psi^{-1} \psi \Longrightarrow \bar{x}^{A} \psi \subseteq \psi \bar{x}^{B}
$$

and

$$
\psi^{-1} x_{*}^{A} \subseteq x_{*}^{B} \Longrightarrow \psi \psi^{-1} x_{*}^{A} \subseteq \psi x_{*}^{B} \Rightarrow x_{*}^{A} \subseteq \psi x_{*}^{B}
$$

However, if $\psi$ is not a mapping, conditions (1) and (2) are not equivalent, as shown by the following

EXAmple.

$$
\begin{aligned}
S_{A} & =\left\{a_{1}, a_{2}, a_{3}, a_{4}\right\}, S_{B}=\left\{b_{1}, b_{2}, b_{3}, b_{4}\right\}, \\
X_{A} & =X_{B}=\{x\}, \\
\bar{x}^{A} & =\left\{\left(a_{1}, a_{4}\right),\left(a_{2}, a_{3}\right)\right\}, \bar{x}^{B}=\left\{\left(b_{1}, b_{4}\right),\left(b_{2}, b_{3}\right)\right\}, \\
\psi & =\left\{\left(a_{1}, b_{1}\right),\left(a_{2}, b_{1}\right),\left(a_{2}, b_{2}\right),\left(a_{3}, b_{3}\right),\left(a_{4}, b_{4}\right)\right\} .
\end{aligned}
$$

In this case condition (1) is satisfied, but condition (2) is not.

Let $\psi$ be a weak homomorphism of $\hat{A}$ onto $\hat{B}$. Then for every input tape $\xi$ we have:

(i) $\psi^{-1} \bar{\xi}^{A} \subseteq \bar{\xi}^{B} \psi^{-1}$,

(ii) $\psi^{-1} \xi_{*}^{A} \subseteq \xi_{*}^{B}$.

Indeed, let $\xi=x_{1} \ldots x_{K}$; then

$$
\psi^{-1} \bar{\xi}^{A}=\psi^{1} \bar{x}_{1}^{A} \ldots \bar{x}_{K}^{A} \subseteq \bar{x}_{1}^{B} \psi^{-1} \bar{x}_{2}^{A} \ldots \bar{x}_{K}^{A} \subseteq \bar{x}_{1}^{B} \ldots \bar{x}_{K}^{B} \psi^{-1}=\bar{\xi}^{B} \psi^{-1}
$$

and

$$
\psi^{-1} \xi_{*}^{A}=\psi^{-1} \bar{x}_{1}^{A} \ldots \bar{x}_{K}^{A}{ }_{1} x_{K *}^{A} \subseteq \bar{x}_{1}^{B} \ldots \bar{x}_{K-1}^{B} \psi^{-1} x_{K *}^{A} \subseteq \bar{x}_{1}^{B} \ldots \bar{x}_{K{ }_{1-1}}^{B} x_{K *}^{B}=\xi_{*}^{B} .
$$

Again, let $\psi$ be a weak homomorphism of $\hat{A}$ onto $\hat{B}$. The relation $\psi$ induces a decomposition $\pi$ of $S_{A}$, namely $\pi=\left\{H_{s}=s \psi^{-1} \mid s \in S_{B}\right\}$. This decomposition $\pi$ is admissible by the semi-automaton $A$ of $\hat{A}$, i.e., for every $H_{s} \in \pi$ and every $x \in X_{A}$ there exists an $H_{t} \in \pi$ such that $H_{s} \bar{x}^{A}$ $\subseteq H_{t}$. Indeed,

$$
H_{s} \bar{x}^{A}=s \psi^{-1} \bar{x}^{A} \subseteq s \bar{x}^{B} \psi^{-1} .
$$

If $s \bar{x}^{B}=t$, we have $H_{s} \bar{x}^{A} \subseteq t \psi^{-1}=H_{t} \in \pi$.

If $s \bar{x}^{B}=\emptyset$, then also $H_{s} \bar{x}^{A}=\emptyset$ and the admissibility condition is trivially satisfied.

Furthermore, $\pi$ is an output-consistent decomposition of $\hat{A}$, i.e., for every $H_{s} \in \pi$ and every tape $\xi, H_{s} \xi_{*}^{A}$ contains at most one element. Indeed,

$$
H_{s} \xi_{*}^{A}=s \psi^{-1} \xi_{*}^{A} \subseteq s \xi_{*}^{B}
$$

and $s \xi_{*}^{B}$ is either the empty set or a single element of $Z_{B}=Z_{A}$.

Conversely, let $\left.\hat{A}=\left\langle S_{A}, X_{A}, Z_{A},\right\lrcorner^{A}, \Lambda^{A}\right\rangle$ be an automaton and $\pi$ an ad- 
missible decomposition of $A$, which is output-consistent in $A$.

Clearly an automaton $\hat{B}=\left\langle\pi, X_{A}, Z_{A}, \Delta^{B}, \Lambda^{B}\right\rangle$ can be defined such that the relation $\psi$ defined by $(s, H) \in \psi \Leftrightarrow s \in H \in \pi$ is a weak homomorphism of $\hat{A}$ onto $\hat{B}$.

The semi-automaton $B$ of $B$ is a $\pi$-factor of $A$.

Summarizing we have the following:

Theorem 1. A weak homomorphism $\psi$ of $\hat{A}$ onto $\hat{B}$ defines naturally an admissible, output-consistent decomposition in $\hat{A}$ and, conversely, every such decomposition in $\hat{A}$ determines at least one corresponding weakly homomorphic image of $\hat{A}$.

Note. In the special case, where $\psi$ is a homomorphism (Definition 1), the decomposition $\pi$ becomes a partition. On the other hand we have the following known

Corollary. An admissible, output-consistent partition of a complete automaton $\hat{A}$ defines naturally a unique homomorphic image of $\hat{A}$.

\section{Covering of automata.}

Definition 3. The automaton $\hat{B}=\left\langle S_{B}, X, Z, \Delta^{B}, \Lambda^{B}\right\rangle$ is said to cover the automaton $\hat{A}=\left\langle S_{A}, X, Z, \Delta^{A}, \Lambda^{A}\right\rangle$ (notation: $\hat{B} \geqq \hat{A}$ ) if there exists a mapping $\chi$ of $S_{A}$ into $S_{B}$ such that for every input tape $\xi$ :

$$
\xi_{*}^{A} \subseteq \chi \xi_{*}^{B} .
$$

Using the relational techniques introduced we now provide a simple proof of the following theorem, which is in fact a modified version of a known result on partial automata [4].

Theorem 2. Let $\psi$ be a weak homomorphism of $\hat{A}$ onto $\hat{B}$. Then $\hat{B} \geqq \hat{A}$.

Proof. Clearly there exists at least one mapping $\chi$ of $S_{A}$ into $S_{B}$, such that $\chi \subseteq \psi$. Using (3) we obtain for every input tape $\xi$ :

$$
\psi^{-1} \xi_{*}^{A} \subseteq \xi_{*}^{B} \Longrightarrow \chi^{-1} \xi_{*}^{A} \subseteq \xi_{*}^{B} \Longrightarrow \chi \chi^{-1} \xi_{*}^{A} \subseteq \chi \xi_{*}^{B} .
$$

But $\mid \chi=S_{A}$; hence $\chi \chi^{-1}$ includes the identity relation $I_{S_{A}}$. Thus we obtain

$$
\xi_{*}^{A} \subseteq \chi \chi^{-1} \xi_{*}^{A} \subseteq \chi \xi_{*}^{B}
$$

In [18] the concept of covering of semi-automata was introduced. Using the notations of this paper we have accordingly:

Definition 4. The semi-automaton $B=\left\langle S_{B}, X, \Delta^{B}\right\rangle$ covers the semiautomaton $A=\left\langle S_{A}, X, \Delta^{A}\right\rangle$ (notation: $B \geqq A$ ) if there exists a mapping $\eta$ of a subset of $S_{B}$ onto $S_{A}$ such that for every $x \in X$

$$
\eta \bar{x}^{A} \subseteq \bar{x}^{B} \eta \text {. }
$$


ThEOREM 3. Let $\hat{A}=\left\langle S_{A}, X, Z, \Delta^{A}, \Lambda^{A}\right\rangle$ be an automaton, and $B=\left\langle S_{B}, X, \Delta^{B}\right\rangle$ a semi-automaton covering the semi-automaton $A$ of $\hat{A}$. Then there exists an automaton $\hat{B}$ with $B$ as its semi-automaton, such that $\hat{B} \geqq \hat{A}$.

Proof. $B \geqq A$ implies the existence of a mapping $\eta$ with $\mid \eta \subseteq S_{B}$ and $\eta \mid=S_{A}$, satisfying (4). Now, let $B=\left\langle S_{B}, X, Z, \Delta^{B}, \Lambda^{B}\right\rangle$ where $\Lambda^{B}$ is defined by:

$$
x_{*}^{B}=\eta x_{*}^{A} \quad \text { for every } x \in X .
$$

In other words $\Lambda^{B}(s, x)=\Lambda^{A}(s \eta, x)$ if $s \in \mid \eta$ and $\Lambda^{A}(s \eta, x)$ is defined. Otherwise $\Lambda^{B}(s, x)$ is not defined.

The automaton $\hat{B}$ covers $\hat{A}$. Indeed, evidently, there exists a mapping $\chi$ of $S_{A}$ into $S_{B}$ such that $\chi \subseteq \eta^{-1}$. Now, for any input tape $\xi=x_{1} x_{2} \cdots x_{K}$

$$
\chi^{-1} \xi_{*}^{A} \subseteq \eta \xi_{*}^{A}=\eta \bar{x}_{1}^{A} \ldots \bar{x}_{K-1}^{A} x_{K *}^{A} \subseteq \bar{x}_{1}^{B} \ldots \bar{x}_{K-1}^{B} \eta x_{K *}^{A}=\bar{x}_{1}^{B} \ldots \bar{x}_{K-1}^{B} x_{K *}^{B}=\xi_{*}^{B} .
$$

Hence

$$
\xi_{*}^{A} \subseteq \chi \chi^{-1} \xi_{*}^{A} \subseteq \chi \xi_{*}^{B}
$$

Theorem 3 is immediately applicable to the direct product of automata, which is defined as follows:

Definition 5. The direct product $\hat{A} \times \hat{B}$ of the automata $\hat{A}=$ $\left\langle S_{A}, X, Z_{A}, \Delta^{A}, \Lambda^{A}\right\rangle$ and $\hat{B}=\left\langle S_{B}, X, Z_{B}, \Delta^{B}, \Lambda^{B}\right\rangle$ is the automaton $\hat{C}=$ $\left\langle S_{C}, X, Z_{C}, \Delta^{C}, \Lambda^{C}\right\rangle$ where

$$
S_{C}=S_{A} \times S_{B}, Z_{C}=Z_{A} \times Z_{B}
$$

and

$$
\begin{aligned}
& \left(s_{A}, s_{B}\right) \bar{x}^{C}=s_{A} \bar{x}^{A} \times s_{B} \bar{x}^{B}, \\
& \left(s_{A}, s_{B}\right) x_{*}^{C}=s_{A} x_{*}^{A} \times s_{B} x_{*}^{B} .
\end{aligned}
$$

Definition 5 implies that $C=A \times B$.

In order to apply Theorem 3 to direct products of automata we shall need the following extension of the covering concept.

Definition 6. The automaton $\hat{B}=\left\langle S_{B}, X, Z_{B}, \Delta^{B}, \Lambda^{B}\right\rangle$ is said to cover widely the automaton $\hat{A}=\left\langle S_{A}, X, Z_{A}, \Delta^{A}, \Lambda^{A}\right\rangle$ (notation: $\hat{B} \geqq \hat{A}$ ), if there exists a mapping $\chi$ of $S_{A}$ into $S_{B}$ and a mapping $\tau$ of a subset of $Z_{B}$ into $Z_{A}$, such that for every input tape $\xi$

$$
\xi_{*}^{A} \subseteq \chi \xi_{*}^{B} \tau
$$

We now have the following

Theorem 4. Let $C$ be the semi-automaton of $\hat{C}=\left\langle S_{C}, X, Z_{C}, د^{C}, \Lambda^{C}\right\rangle$ and $A, B$ semi-automata such that $A \times B \geqq C$. Then there exist automata $\hat{A}, \hat{B}$ with $A$ and $B$ as semi-automata, respectively, such that

$$
\hat{A} \times \hat{B} \geqq \hat{C} .
$$


Proof. By Theorem 3 there exists an automaton $D$ such that $D=A \times B$ and $D \geqq C$.

We define $Z_{A}=S_{A} \times X$ and $Z_{B}=S_{B} \times X$. Next, we define $\Lambda^{A}$. If for a given state $s_{A} \in S_{A}$ and a given input $x \in X$, there exists an $s_{B} \in S_{B}$, such that $\left(s_{A}, s_{B}\right) x_{*}^{D} \neq \emptyset$, let $s_{A} x_{*}^{A}=\left(s_{A}, x\right)$. Otherwise $s_{A} x_{*}^{A}=\emptyset$.

$\Lambda^{B}$ is defined in an analogous way.

Introducing the map $\tau$ by

$$
\left(s_{A}, s_{B}\right) x_{*}^{D} \neq \emptyset \Longrightarrow\left(\left(s_{A}, x\right),\left(s_{B}, x\right)\right) \tau=\left(s_{A}, s_{B}\right) x_{*}^{D},
$$

we obtain

$$
\xi_{*}^{D}=\xi_{*}^{E} \tau,
$$

where

$$
\hat{E}=\hat{A} \times \hat{B} \text {. }
$$

Let $\chi$ be the mapping corresponding to the covering $D \geqq C$; then

$$
\xi_{*}^{C} \subseteq \chi \xi_{*}^{D}=\chi \xi_{*}^{E} \tau, \text { i.e., } \hat{E}=\hat{A} \times \hat{B} \geqq \hat{C} .
$$

Evidently the above discussion of direct products of two automata can be extended to any finite number of automata.

IV. Cascade products of automata. The following definition generalizes the concept of cascade connection in [16].

Definition 7. Let $\hat{A}=\left\langle S_{A}, X_{A}, Z_{A}, \Delta^{A}, \Lambda^{A}\right\rangle$ and $\hat{B}=\left\langle S_{B}, X_{B}, Z_{B}, \Delta^{B}, \Lambda^{B}\right\rangle$ be automata with $Z_{A} \subseteq X_{B}$. The cascade product $\hat{C}=\hat{A} \circ \hat{B}$ is defined as the automaton $C=\left\langle S_{C}, X_{C}, Z_{C}, \Delta^{C}, \Lambda^{C}\right\rangle$ where $S_{C}=S_{A} \times S_{B}, X_{C}=X_{A}, Z_{C}$ $=Z_{B}$ and for every $x \in X_{C}$

$$
\begin{aligned}
& \left(s_{A}, s_{B}\right) \bar{x}^{C}=s_{A} \bar{x}^{A} \times s_{B} \bar{y}^{B}, \\
& \left(s_{A}, s_{B}\right) x_{*}^{C}=s_{B} y_{*}^{B},
\end{aligned}
$$

where

$$
Z_{A} \ni y=s_{A} x_{*}^{A} .
$$

(If $y=\emptyset$, then $\bar{y}$ and $y_{*}$ is to be understood as $\emptyset$.)

Definition 7 is naturally modified to the case $\hat{A} \circ B$, where $B$ is a semiautomaton. We thus obtain

$$
\hat{C}=\hat{A} \circ \hat{B} \Rightarrow C=\hat{A} \circ B .
$$

With any semi-automaton $A=\left\langle S_{A}, X_{A}, \Delta^{A}\right\rangle$ one may associate the automaton $A^{*}=\left\langle S_{A}, X_{A}, Z_{A}, \Delta^{A}, \Lambda^{A}\right\rangle$ where $Z_{A}=S_{A} \times X_{A}$ and $s_{A} x_{*}^{A}=\left(s_{A}, x\right)$ for every $s_{A} \in S_{A}$ and every $x \in X_{A}$. If $B=\left\langle S_{B}, X_{B}, \Delta^{B}\right\rangle$ is a semi-automaton, such that $X_{B} \supseteq S_{A} \times X_{A}$, we define (following [18]):

$$
A \circ B=A^{*} \circ B \text {. }
$$


We now prove

THEOREM 5. Let $\hat{A}$ and $\hat{C}$ be automata and $B$ a semi-automaton such that $\hat{A} \circ B \geqq C$. Then there exist automata $\hat{D}$ and $\hat{E}$ such that $\hat{D} \circ \hat{E} \geqq C$, where $D=A, \hat{D} \geqq \hat{A}, S_{E}=S_{B}$.

Proof. By Theorem 3 there exists an automaton $\hat{F}$ such that $F=\hat{A} \circ B$ and $\hat{F} \geqq C$. We now define $D$ as follows:

$D=A, Z_{D}=S_{D} \times X_{D}$, and for every $s_{D} \in S_{D}$ and every $x \in X_{D}, s_{D} x_{*}^{D}$ $=\left(s_{D}, x\right)$.

Introducing the map $\tau$ of $Z_{D}$ into $Z_{A}$ determined by $\left(s_{D}, x\right) \tau=s_{D} x_{*}^{A}$ we clearly obtain:

$$
\hat{D} \geqq \hat{A}
$$

Next, we define $\hat{E}$ as follows:

$$
S_{E}=S_{B}, X_{E}=Z_{D}, Z_{E}=Z_{F}
$$

for every $\left(s_{D}, x\right) \in X_{E}$ and every $s_{E} \in S_{E}$

$$
\begin{gathered}
\overline{\left(s_{D}, x\right)}{ }^{E}=\overline{\left(s_{D}, x\right) \tau^{B},} \\
s_{E}\left(s_{D}, x\right)_{*}^{E}=\left(s_{D}, s_{E}\right) x_{*}^{F} .
\end{gathered}
$$

Denoting $\hat{G}=\hat{D} \circ \hat{E}$, we proceed to show that $\hat{F}=\hat{G}$. Indeed:

$$
\begin{aligned}
& S_{F}=S_{A} \times S_{B}=S_{D} \times S_{E}=S_{G}, \\
& X_{F}=X_{A}=X_{D}=X_{G}, Z_{F}=Z_{E}=Z_{G},
\end{aligned}
$$

and

$$
s_{G} \bar{x}^{G}=\left(s_{D}, s_{E}\right) \bar{x}^{G}=s_{D} \bar{x}^{D} \times s_{E} \bar{w}^{E}
$$

where

$$
w=s_{D} x_{*}^{D}=\left(s_{D}, x\right)
$$

Now

$$
\bar{w}^{E}={\overline{\left(s_{D}, x\right)}}^{E}={\overline{\left(s_{D}, x\right)} \tau^{B}}^{B}=\bar{y}^{B}
$$

where

$$
y=\left(s_{D}, x\right) \tau=s_{D} x_{*}^{A} .
$$

Hence $s_{G} \bar{x}^{(i}=s_{D} \bar{x}^{D} \times s_{E} \bar{w}^{E}=s_{D} \bar{x}^{A} \times s_{E} \bar{y}^{B}=\left(s_{D}, s_{E}\right) \bar{x}^{F}=s_{G} \bar{x}^{F}$.

Furthermore,

$$
s_{G} x_{*}^{G}=\left(s_{D}, s_{E}\right) x_{*}^{G}=s_{E} w_{*}^{E}=s_{E}\left(s_{D}, x\right)_{*}^{E}=\left(s_{D}, s_{E}\right) x_{*}^{F}=s_{G} x_{*}^{F} .
$$

Thus $\hat{D} \circ \hat{E}=\hat{G}=\hat{F} \geqq \hat{C}$. 
Referring to the definition of cascade-product of semi-automata, we obtain the following:

Corollary. Given an automaton $\hat{C}$ and two semi-automata $A, B$ such that $A \circ B \geqq C$ then there exist automata $\hat{A}, \hat{B}$ such that $\hat{A} \circ \hat{B} \geqq C$, where $A, B$ are the semi-automata of $\hat{A}, \hat{B}$, respectively.

In particular cases the automaton $\hat{D}$ required in Theorem 5 can be constructed with a reduced output set (cf. [17]).

We now restrict our considerations to complete, finite automata. For such an automaton $\hat{A}$ we denote by $\# A$ the number of its states. If $\pi$ is a decomposition of the finite set $S,|\pi|$ will denote the number of elements in the largest class of $\pi$.

The following result was obtained in [18]:

Theorem 6. Let $A=\left\langle S_{A}, X_{A}, \Delta^{A}\right\rangle$ be a complete, finite semi-automaton, $\pi$ an admissible decomposition of $A$, and $B$ a $\pi$-factor of $A$. Then there exist semi-automata $C$ and $D$, such that $C$ is isomorphic to $B, \# D=|\pi|$, and $C \circ D \geqq A$.

We now prove

Theorem 7. Let $\hat{A}, \hat{B}, \hat{C}$ be complete, finite automata, such that there exists a homomorphism $\phi$ from $\hat{A} \circ \hat{B}$ onto $\hat{C}$. Then there exist complete, finite automata $\hat{D}, \hat{E}$ and an admissible decomposition $\beta$ of $C$ such that $D$ is isomorphic to a $\beta$-factor of $C$ and

$$
\hat{D} \circ \hat{E} \geqq \hat{C}, \# D \leqq \# A, \# E \leqq \# .
$$

Proof. Denote $\hat{A} \circ \hat{B}=\hat{F}$, and let $\rho$ be the admissible partition of $F$ determined by

$$
\left(s_{A}, s_{B}\right) \equiv\left(s_{A}^{\prime}, s_{B}^{\prime}\right)(\rho) \Leftrightarrow s_{A}=s_{A}^{\prime} .
$$

Then the unique $\rho$-factor $F / \rho$ is isomorphic to $A(F / \rho \cong A)$.

Let $\pi$ be the natural partition of $F$ determined by $\phi$. Then $F / \pi \cong C$. We now consider the decomposition $\alpha$ of $\pi$ defined by:

$$
\alpha=\left\{\alpha_{H}=\{\pi(s) \mid s \in H\} \mid H \in \rho\right\}
$$

where $\pi(s)$ denotes the class of $\pi$ containing $s$.

$\alpha$ is admissible by the semi-automaton $F / \pi$. Indeed, let $\alpha_{1} \in \alpha$, i.e., there exists an $H \in \rho$, such that $\alpha_{1}=\{\pi(s) \mid s \in H\}$.

For any input $x$ of $F$ we have

$\alpha_{1} \bar{x}^{F / \pi}=\{\pi(s) \mid s \in H\} \bar{x}^{F / \pi}=\left\{\pi\left(s \bar{x}^{F}\right) \mid s \in H\right\} \subseteq\left\{\pi(t) \mid t \in H \bar{x}^{F / \rho} \in \rho\right\} \in \alpha$.

Furthermore, \# $\alpha \leqq \# \rho=\# A$.

For every $H \in \rho, \# \alpha_{H} \leqq \# H \leqq|\rho|$. 
Hence $|\alpha| \leqq|\rho|=\# B$.

The isomorphism $C \cong F / \pi$ implies the existence of an admissible decomposition $\beta$ of $C$ corresponding to the decomposition $\alpha$ of $F / \pi$.

We thus have

$$
\# \beta \leqq \# A,|\beta| \leqq \# B .
$$

Now we apply Theorem 6 to obtain semi-automata $D, E$ such that $D \circ E \geqq C$, where $D$ is isomorphic to a $\beta$-factor of $C$, and $\# E=|\beta| \leqq \# B$.

$D$ being isomorphic to a $\beta$-factor of $C$, satisfies $\# D=\# \beta \leqq \# A$.

As final step of this proof we apply the Corollary to Theorem 5 and obtain automata $D$ and $E$ such that $D \circ \hat{E} \geqq C$ with $D, E$ as semiautomata of $D, E$ respectively.

J. Hartmanis and R. E. Stearns have pointed out in [9] that state reduction may destroy possibilities of cascade decompositions. Our Theorem 7 shows that even after state reduction of a complete automaton, i.e., its replacement by a homomorphic image, the original possibilities for cascade decompositions may be reconstructed in a certain sense.

$W_{\epsilon}$ shall illustrate this point by using the example in [9].

Let $\hat{A}, \hat{B}, C$ be the automata given by Tables I, II, III respectively:

Table I $-\hat{A}$

input

$0 \quad 1$

\begin{tabular}{|c|c|c|}
\hline$a_{1}$ & $a_{1}^{\prime} y_{1}$ & $a_{2} / y_{2}$ \\
\hline$a_{2}$ & $a_{1} / y_{3}$ & $a_{1} / y_{4}$ \\
\hline
\end{tabular}

next state/output

Table II $-\hat{B}$

input

\begin{tabular}{cc|c|c|c|c|c|}
\multicolumn{1}{c}{} & \multicolumn{2}{c}{$y_{1}$} & \multicolumn{1}{c}{$y_{2}$} & \multicolumn{1}{c}{$y_{3}$} & \multicolumn{1}{c}{$y_{4}$} & output \\
& $b_{1}$ & $b_{3}$ & $b_{3}$ & $b_{2}$ & $b_{4}$ & 1 \\
\hline \multirow{2}{*}{ state } & $b_{i}$ & $b_{4}$ & $b_{4}$ & $b_{1}$ & $b_{3}$ & 0 \\
\cline { 2 - 7 } & $b_{3}$ & $b_{1}$ & $b_{2}$ & $b_{4}$ & $b_{4}$ & 0 \\
\hline & $b_{4}$ & $b_{2}$ & $b_{1}$ & $b_{3}$ & $b_{3}$ & 1 \\
\hline
\end{tabular}

next state 
Table III $-\hat{C}$

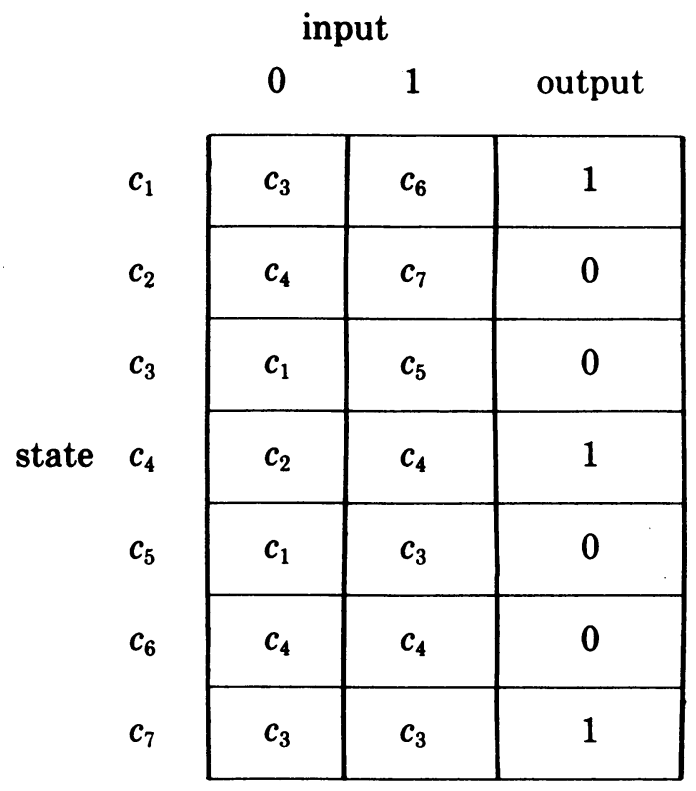

next state

The automaton $\hat{F}=\hat{A} \circ \hat{B}$ is given in Table IV.

\begin{tabular}{|c|c|c|c|}
\hline & able & & \\
\hline & 0 & 1 & output \\
\hline$\left(a_{1}, b_{1}\right)=f_{1}$ & $f_{3}$ & $f_{7}$ & 1 \\
\hline$\left(a_{1}, b_{2}\right)=f_{2}$ & $f_{4}$ & $f_{8}$ & 0 \\
\hline$\left(a_{1}, b_{3}\right)=f_{3}$ & $f_{1}$ & $f_{6}$ & 0 \\
\hline$\left(a_{1}, b_{4}\right)=f_{4}$ & $f_{2}$ & $f_{5}$ & 1 \\
\hline$\left(a_{2}, b_{1}\right)=f_{5}$ & $f_{2}$ & $f_{4}$ & 1 \\
\hline$\left(a_{2}, b_{2}\right)=f_{6}$ & $f_{1}$ & $f_{3}$ & 0 \\
\hline$\left(a_{2}, b_{3}\right)=f_{7}$ & $f_{4}$ & $f_{4}$ & 0 \\
\hline$\left(a_{2}, b_{4}\right)=f_{8}$ & $f_{3}$ & $f_{3}$ & 1 \\
\hline
\end{tabular}


The automata $\hat{F}$ and $\hat{C}$ are identical with machines $B^{*}$ and $B$ of Figure 4 in [9].

The mapping

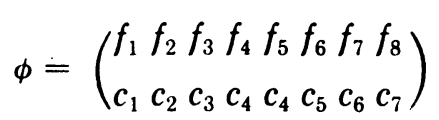

is a homomorphism of $\hat{F}$ onto $\hat{C}$.

Indeed,

$$
\begin{gathered}
\overline{0}_{\phi}^{F}=\left(\begin{array}{llllllll}
f_{1} & f_{2} & f_{3} & f_{4} & f_{5} & f_{6} & f_{7} & f_{8} \\
c_{3} & c_{4} & c_{1} & c_{2} & c_{2} & c_{1} & c_{4} & c_{3}
\end{array}\right)=\phi \overline{0}^{c}, \\
0_{*}^{F}=\left(\begin{array}{llllllll}
f_{1} & f_{2} & f_{3} & f_{4} & f_{5} & f_{6} & f_{7} & f_{8} \\
1 & 0 & 0 & 1 & 1 & 0 & 0 & 1
\end{array}\right)=\phi 0_{*}^{C},
\end{gathered}
$$

and similarly for the input 1 .

The partitions $\rho$ and $\pi$ of $F$ become:

$$
\begin{aligned}
& \rho=\left\{H_{1}=\left\{f_{1}, f_{2}, f_{:}, f_{4}\right\}, H_{2}=\left\{f_{5}, f_{6}, f_{7}, f_{8}\right\}\right\}, \\
& \pi=\left\{\pi_{1}=\left\{f_{1}\right\}, \pi_{2}=\left\{f_{2}\right\}, \pi_{3}=\left\{f_{3}\right\},\right. \\
& \left.\pi_{4}=\left\{f_{4}, f_{5}\right\}, \pi_{5}=\left\{f_{6}\right\}, \pi_{6}=\left\{f_{7}\right\}, \pi_{7}=\left\{f_{8}\right\}\right\} .
\end{aligned}
$$

The decomposition $\alpha$ of $\pi$ becomes:

$$
\alpha=\left\{\alpha_{1}=\left\{\pi_{1}, \pi_{2}, \pi_{3}, \pi_{4}\right\}, \alpha_{2}=\left\{\pi_{4}, \pi_{5}, \pi_{6}, \pi_{7}\right\}\right\} .
$$

The decomposition $\beta$ of $C$ is therefore

$$
\beta=\left\{\beta_{1}=\left\{c_{1}, c_{2}, c_{3}, c_{4}\right\}, \beta_{2}=\left\{c_{4}, c_{5}, c_{6}, c_{7}\right\}\right\} .
$$

Let $D$ be the $\beta$-factor of $C$ given in Table $\mathrm{V}$.

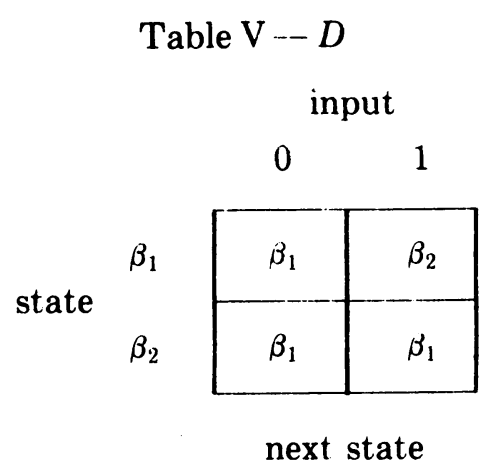

The application of Theorem 6 leads to a semi-automaton $E$ isomorphic to $B$.

Using the construction of Theorem 5 we obtain automata $\hat{D}$ and $\hat{E}$ iso- 
morphic with $\hat{A}$ and $\hat{B}$ respectively and $\hat{D} \circ \hat{E} \geqq \hat{C}$.

On the other hand, as shown in [9], the usual methods of cascadedecomposition applied to $C$ do not lead to the discovery of the above cascade-decomposition of $\hat{C}$.

Conclusion. This paper shows the convenience of the relational techniques introduced for the study of automata.

Furthermore, a generalized approach to the problem of automata decomposition has been developed. Namely, in order to synthesize $\hat{A}$, it is frequently convenient to construct a cover of $\hat{A}$ which is a direct or cascade product of simpler automata. (The previous approach was restricted to equality or inclusion instead of covering.)

It appears that the results obtained in this paper will have actual engineering applications to the synthesis of sequential machines.

However, in order to extend this applicability, the development of an efficient technique for obtaining suitable admissible decompositions of automata is required.

\section{REFERENCES}

1. A. Gill, Cascaded finite-state machines, IRE Trans. Electronic Computers EC-10 (1961), 366-370.

2. Introduction to the theory of finite-state machines, McG raw-Hill, New York, 1962.

3. S. Ginsburg, Some remarks on abstract machines, Trans. Amer. Math. Soc. 96 (1960), 400-444.

4. _ An introduction to mathematical machine theory, Addison-Wesley, Reading, Mass., 1962.

5. V. M. Gluškov, Abstraktnaya teoriya avtomatov, Uspehi Mat. Nauk 16 (1961), 3-62.

6. Sintez tsifrovyh avtomatov, Gos. Izdat. Fiz. Mat. Lit., Moscow, 1962.

7. J. Hartmanis, Symbolic analysis of a decomposition of information processing machines, Information and Control 3 (1960), 154-178.

8. L Loop-free structure of sequential machines, Information and Control 5 (1962), 25-43.

9. J. Hartmanis and R. E. Stearns, Some dangers in state reduction of sequential machines, Information and Control 5 (1962), 252-260.

10. S. Huzino, Theory of finite automata, Mem. Fac. Sci. Kyushu Univ. Ser. A 15 (1962), 95-159.

11. K. B. Krohn and J. L. Rhodes, Algebraic theory of machines, Proc. Sympos. on Math. Theory of Automata, New York, 1962, pp. 341-384, Wiley, New York, 1963.

12. G. H. Mealy, A method for synthesizing sequential circuits, Bell System Tech. J. 34 (1955), 1045-1079.

13. E. F. Moore (editor), Sequential machines: Selected papers, Addison-Wesley, Reading, Mass., 1964.

14. Polytechnic Institute of Brooklyn, Mathematical theory of automata, Proc. Sympos. on Math. Theory of Automata, New York, 1962, Wiley, New York, 1963.

15. D. Tamari and A. Ginzburg, Representation of multiplicative systems by families of binary relations (I), J. London Math. Soc. 37 (1962), 410-423.

16. M. Yoeli, The cascade decomposition of sequential machines, IRE Trans. Electronic Computers EC-10 (1961), 587-592.

17. _— Cascade-parallel decompositions of sequential machines, IEEE Trans. Elec- 
tronic Computers EC-12 (1963), 322-324.

18. , Decompositions of finite automata, Tech. Rep. No. 10, U. S. Office of Naval Research, Information Systems Branch, (1963); Hebrew University, Jerusalem, lsrael, DDC Document AD-406302.

19. - Multi-valued homomorphic mappings and subdirect covers of partial algebras, Tech. Summary Rep. No. 493, Math. Research Center, U. S. Army, Madison, Wisconsin, 1964, DDC Document AD-605649.

Technion, IsRael Institute of Technology, HaIfa, IsRaEl

STANFORd RESEARCH INSTITUTE, Menlo Park, California 